\title{
Polysynaptic Potentiation at Different Levels of Rat Olfactory Pathways Following Learning
}

\author{
Anne Marie Mouly ${ }^{1}$ and Rémi Gervais \\ Institut des Sciences Cognitives, Centre National de la Recherche Scientifique Unité Mixte de Recherche (UMR) 5015, 69675 Bron Cédex, France
}

\begin{abstract}
This study was aimed at investigating the consequences of learning on late polysynaptic components of evoked field potential signals recorded in parallel at different levels of the olfactory pathways. For this, evoked field potentials induced by electrical stimulation of the olfactory bulb were recorded simultaneously in the anterior piriform cortex, the posterior piriform cortex, the lateral entorhinal cortex, and the dentate gyrus. The different parameters of late components were measured in each site before and after completion of associative learning in anesthetized rats. In the learning task, rats were trained to associate electrical stimulation of one olfactory bulb electrode with the delivery of sucrose (positive reward) and stimulation of a second olfactory bulb electrode with the delivery of quinine (negative reward). In this way, stimulation of the same olfactory bulb electrodes used for inducing field potentials served as a discriminative cue in the learning paradigm. The data confirmed previous observation that learning was associated with a lowering in late-component-1 intensity of induction in the posterior piriform cortex. The use of simultaneous recording allowed us to further specify the consequences of learning on late-component distribution in the studied network. Indeed the data showed that whereas before learning, late component 1 was rather uniformly distributed among the recorded sites; following learning, its expression was facilitated preferentially in the posterior piriform cortex and lateral entorhinal cortex. Furthermore, learning was accompanied by the emergence of a new late component (late component 2), which occurred simultaneously in the four recording sites. The possible involvement of potentiation of polysynaptic components in recognition and/or consolidation processes will be discussed.
\end{abstract}

In rodents, olfaction plays a dominant role in the control of behavior. Moreover rats display remarkable facility for learning many complex odor-guided tasks (Otto and Eichenbaum 1992; Slotnick 2001). In addition, the relatively simple anatomy of the olfactory system of rats and the existence of direct projections from the olfactory cortex to the hippocampal formation has led to the suggestion that the rodent olfactory system provides a fruitful domain in which to investigate the neurobiology of memory (Otto and Eichenbaum 1992).

The common view of memory supports the idea that long-term memory is the result of long-lasting changes in synaptic efficacy throughout the neuronal network that includes limbic, as well as sensory cortical areas. However, this view has been supported through studies recording from only one brain structure, hindering the understanding of how brain areas within the network may be simultaneously altered with learning (for review, see Martin et al. 2000). The present study was aimed at investigating learning-induced changes simultaneously at several levels of the olfactory pathways, using changes in evoked field potential signal as a marker of plasticity.

${ }^{1}$ Corresponding author.

E-MAIL mouly@isc.cnrs.fr; FAX (33) 043-7911210.

Article and publication are at http://www.learnmem.org/cgi/doi/ $10.1101 / \mathrm{lm} .45602$
Field potentials have been a useful tool in understanding the plasticity underlying learning. In our study, field potential signals induced by electrical stimulation of the olfactory bulb (OB) were recorded simultaneously in the piriform cortex (anterior part, aPC; posterior part, pPC), the lateral entorhinal cortex (LEC), and the dentate gyrus (DG) before and after associative olfactory learning acquisition. In the learning task, electrical stimulation of the $\mathrm{OB}$ was used to mimic an olfactory stimulus (Mouly et al. 1985, 2001). One of the main advantages of this paradigm was that stimulation of the same OB electrodes used for inducing field potentials also served as a discriminative cue in the learning task.

The OB stimulation evoked field potential is mainly composed of an early component followed in certain conditions of stimulation by a late component corresponding to a massive synchronous discharge of underlying neuronal population (Mouly et al. 1998). In a recent study using the same technique and learning paradigm as described above, we studied the evolution of the early component of the local field potential in the different recording sites (Mouly et al. 2001). We showed that learning was associated with a long-lasting potentiation of this early component in the pPC and LEC. In our present work, we focused on the late component of the evoked field potential signal for the following reasons. First, Stripling et al. (1988) and Stripling and Patneau (1999) have shown that repeated high-frequency

LEARNING \& MEMORY 9:66-75 @ 2002 by Cold Spring Harbor Laboratory Press ISSN1072-0502/02 \$5.00

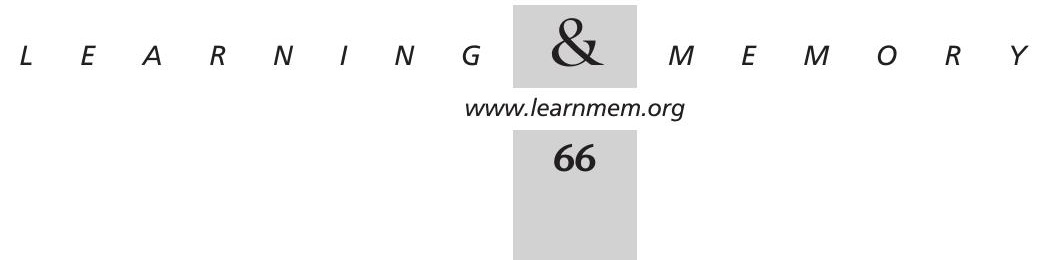


stimulation of the granule cell layer of the OB was able to induce a selective potentiation of late component in field potentials evoked in piriform cortex without altering the early component. Second, in a previous work using voltagesensitive dye to map piriform cortex activity, we showed that olfactory learning greatly enhanced the occurrence of a late component posterior piriform cortex (Litaudon et al. 1997). Finally, Chaillan et al. (1999) reported that olfactory associative learning was accompanied by potentiation of a late polysynaptic response recorded in the DG in response to electrical stimulation of the lateral olfactory tract. Taken together these data suggest that potentiation of polysynaptic components could be induced in parallel at several levels of the olfactory pathways during learning. The present work addressed this question using simultaneous recording of late component in field potentials collected in the same animals in the different structures.

\section{RESULTS}

\section{General Characteristics of Late vs. Early Components}

In both control and trained groups, we measured prelearning amplitude and latency of early and late components in the different recording sites in order to define the general characteristics of these two components before learning. One-way analysis of variance (ANOVA) on the late component amplitude revealed a significant global effect of the recording site $\left(F_{3,27}=11.8 ; P<0.001\right)$. Further comparisons showed that late-component amplitude was greater in the pPC than in any of the other three sites (Wilcoxon tests; $P<0.005$; Table 1). Concerning latency of the late component, one-way ANOVA followed by Wilcoxon comparisons showed that the late-component latency was lower in the pPC than in any of the other three sites $\left(F_{3,27}=20.19\right.$; $P<0.001$; Wilcoxon tests, $P<0.01$; Table 1$)$. In addition latency in the DG was higher than in aPC and LEC $(P<0.01)$.

Similarly to the late component, the early-component amplitude also showed a significant global effect on recording site as indicated by a one-way ANOVA $\left(F_{3,42}=21.2\right.$; $P<0.001)$. However, further comparisons showed that early-component amplitude was maximal in aPC and showed a significant decrease from rostral to caudal sites (Wilcoxon tests, $P<0.005$; Table 1 ). In parallel, latency of the early component was minimal in aPC and increased significantly from aPC to LEC $\left(F_{3,42}=271 ; P<0.001\right.$; Wilcoxon tests, $P<0.005$; Table 1$)$. Additional Wilcoxon comparisons carried out for each recording site between earlyand late-component amplitudes revealed that in all the recorded sites except aPC, the late-component amplitude was greater than (pPC, $P=0.01$ ) or at least not different from (LEC, $P=0.5$; DG, $P=0.4$ ) early-component amplitude.

In summary, whereas the early component presented the greatest amplitude and occurred with the shortest latency in the aPC; the late component presented the greatest amplitude and occurred with the shortest latency in the pPC. Except for aPC, late-component amplitude was at least equal to early-component amplitude.

\section{Effect of Learning on Late-Component Optimal Intensity}

The late-component optimal intensity was measured in pPC signals before and after learning in both the trained and control group. In the trained group, two-way (learning $\times$ reinforcement) ANOVA for repeated measures showed a significant effect of learning $\left(F_{1,19}=7.09 ; P=0.015\right)$ but no effect of reinforcement $\left(F_{1,19}=0.044 ; P=0.837\right)$ or of learning $\times$ reinforcement interaction $\quad\left(F_{1,19}=0.238\right.$; $P=0.632)$. Therefore, $\mathrm{S}+$ and $\mathrm{S}-$ signals were pooled for further analysis. Wilcoxon comparisons showed that learning induced a significant lowering in late-component optimal intensity value $(-33 \%$; mean value before learning: $212 \pm 21 \mu \mathrm{A} ;$ mean value after learning: $141 \pm 24 \mu \mathrm{A}$; $P=0.013$ ) (Fig. 1A). This effect is illustrated in Figure 1B and represents the signals collected in the same animal before and after learning. Specifically, although the late component was not present before learning, it was clearly observable after learning using the same intensity of stimula-

Table 1. Amplitude and Latency of Early and Late components in the Different Recording Sites in Naive Animals

\begin{tabular}{lccccc}
\hline & & \multicolumn{4}{c}{ Structure } \\
\cline { 3 - 6 } Component & Parameter & $\begin{array}{l}\text { Anterior part of } \\
\text { piriform cortex }\end{array}$ & $\begin{array}{c}\text { Posterior part of } \\
\text { piriform cortex }\end{array}$ & $\begin{array}{c}\text { Lateral entorhinal } \\
\text { cortex }\end{array}$ & Dentate gyrus \\
\hline \multirow{2}{*}{ Late } & Amplitude $(\mu \mathrm{V})$ & $296 \pm 82$ & $616 \pm 63^{\mathrm{b}}$ & $262 \pm 52$ & $333 \pm 59$ \\
\multirow{2}{*}{ Early } & Latency $(\mathrm{msec})$ & $58.9 \pm 2.1$ & $54.7 \pm 1.6^{\mathrm{a}}$ & $59.2 \pm 1.7$ & $63.3 \pm 1.2^{\mathrm{a}}$ \\
& Amplitude $(\mu \mathrm{V})$ & $642 \pm 74^{\mathrm{b}, \mathrm{d}}$ & $408 \pm 27^{\mathrm{b}, \mathrm{c}}$ & $194 \pm 18^{\mathrm{b}}$ & $286 \pm 33^{\mathrm{b}}$ \\
& Latency $(\mathrm{msec})$ & $7.7 \pm 0.1^{\mathrm{b}}$ & $9.7 \pm 0.1^{\mathrm{b}}$ & $12.4 \pm 0.2^{\mathrm{b}}$ & $35.8 \pm 1.3^{\mathrm{b}}$ \\
\hline
\end{tabular}

${ }^{\mathrm{a}} P<0.01,{ }^{\mathrm{b}} P<0.005$, significant difference with the values in the same $\mathrm{rcw}$; ${ }^{\mathrm{c}} P<0.01,{ }^{\mathrm{d}} P<0.005$ : significant difference with late-component amplitude in the same column.

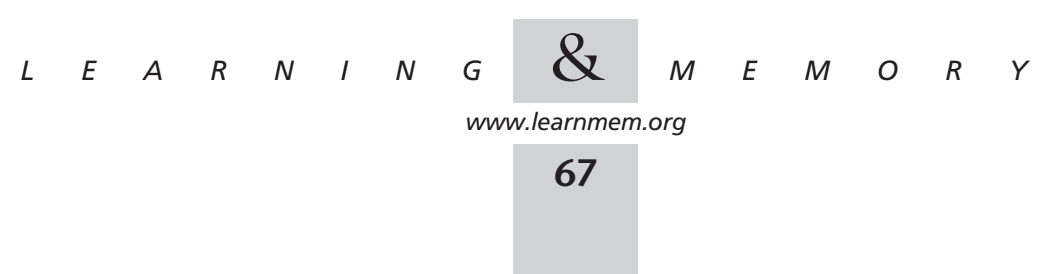


A

\section{Intensity in $\mu \mathrm{A}$}

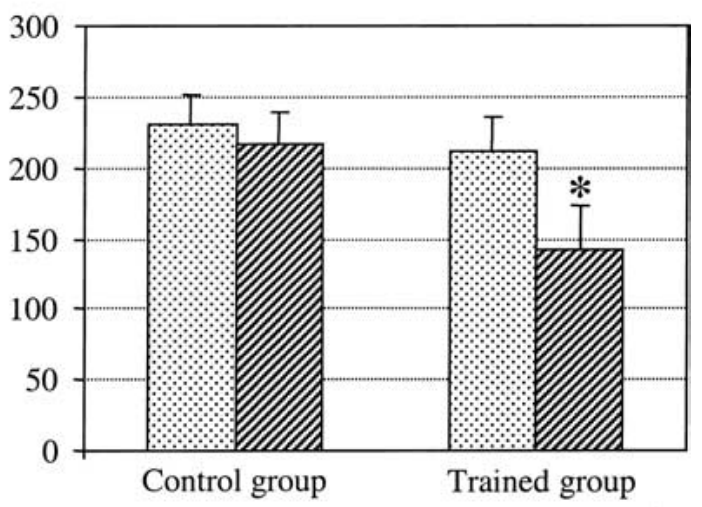

Before learning $\mathbb{Z}$ After learning

B

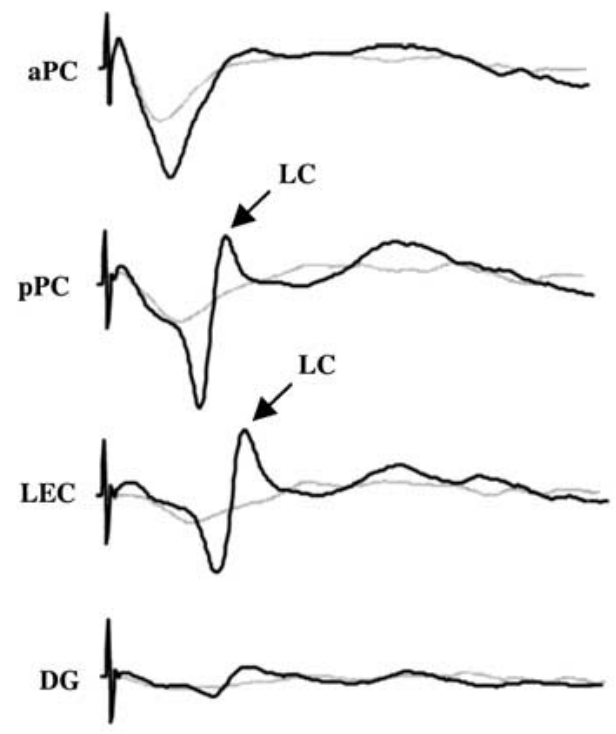

$0.5 \mathrm{mV} \frac{\mid}{20 \mathrm{~ms}}$

Figure 1 Effect of learning on late-component optimal intensity of occurrence. (A) Comparison of the mean value $( \pm$ S.E.M. $)$ of the optimal intensity of the late component in the posterior piriform cortex (pPC) before and after learning in the control group $(n=7)$ and in the trained group $(n=11)$. Learning induces a significant decrease in optimal intensity mean value in trained animals (asterisk, $P=0.013$, Wilcoxon Test). (B) An example of individual evoked field potentials (traces are averages of 12 sweeps) collected in a trained rat in the different sites before (light grey line) and after (dark line) learning for the same intensity of stimulation of the olfactory bulb. Following learning, the late component (LC) was clearly observed in the pPC and the lateral entorhinal cortex, whereas it was absent in the same sites before learning. tion. It is noteworthy that for these low intensities of stimulation, early component was barely detectable.

In control animals, two-way ANOVA for repeated measures revealed no significant effect of the repeated stimulation $\left(F_{1,19}=0.008 ; P=0.93\right)$, the stimulation pattern $\left(F_{1,19}=0.522 ; P=0.487\right)$, or their interaction $\left(F_{1,19}=0.002\right.$; $P=0.968)$ on late-component optimal intensity value (mean value before learning: $230 \pm 23 \mu \mathrm{A}$; mean value after: $236 \pm 32 \mu \mathrm{A}$ ). Thus, in comparison to controls, learning was accompanied by a significant lowering of late-component optimal intensity of stimulation as assessed in pPC.

\section{Effect of Learning on Late-Component Occurrence}

The late component's rate of occurrence was measured in the two experimental groups. First, a comparison was made on signals collected after learning for each recording site between control and trained animals, using the lowered intensity of stimulation. The data are presented in Figure 2A. Chi-square comparisons revealed that the late-component occurrence rate was significantly increased in pPC $(P=0.05)$ and LEC $(P=0.008)$ of trained rats compared to control rats. The late-component rate of occurrence was then compared between the different recording sites in animals of the trained group either before or after learning, using the optimal intensity of stimulation (Fig. 2B). The data show that whereas before learning, the late-component rate of occurrence was not significantly different between the recorded sites; after learning it was significantly higher in pPC $(P<0.005$, Fisher's test $)$ and LEC $(P<0.025$, Fisher's test) than in the remaining two sites. Further comparisons showed that the latter observation can also be explained by a decrease in the late-component rate of occurrence in aPC and DG after learning compared to before learning $(P<$ 0.005).

In summary, in addition to lowering the threshold of occurrence of the late component, learning has also changed its distribution in the network. Following learning, the late component occurring for the lowered intensity of stimulation, is observed preferentially in the pPC and LEC.

\section{Effect of Learning on Late-Component Amplitude}

The amplitude of the late component recorded after learning in PPC and LEC was measured and compared to that obtained before learning using one-way ANOVA for repeated measures, followed by Wilcoxon comparisons. No significant change in the late-component amplitude was observed in pPC or LEC signals following learning (Table 2). Similarly, in control animals, the late-component amplitude in PPC and LEC remained stable after the stimulation paradigm. Furthermore, the late-component amplitude was al-

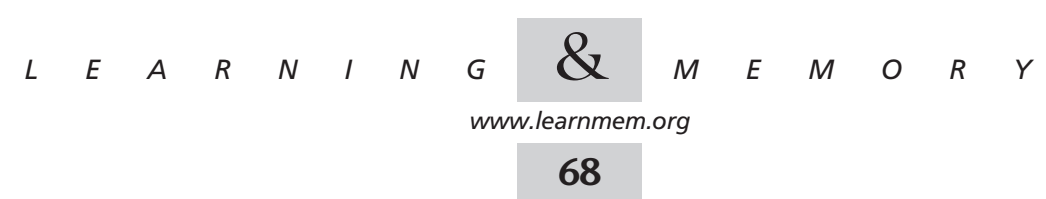


A

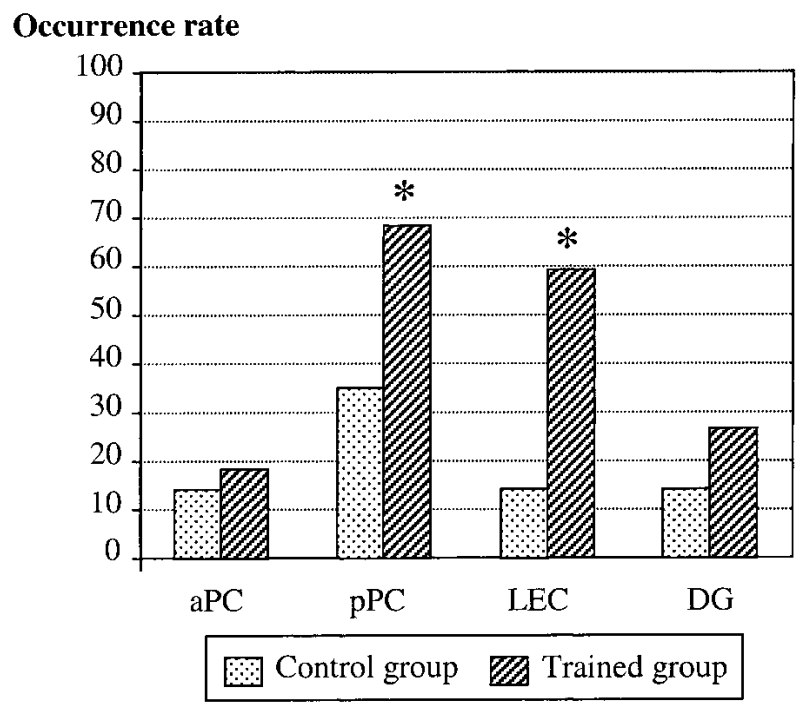

B

\section{Occurrence rate}

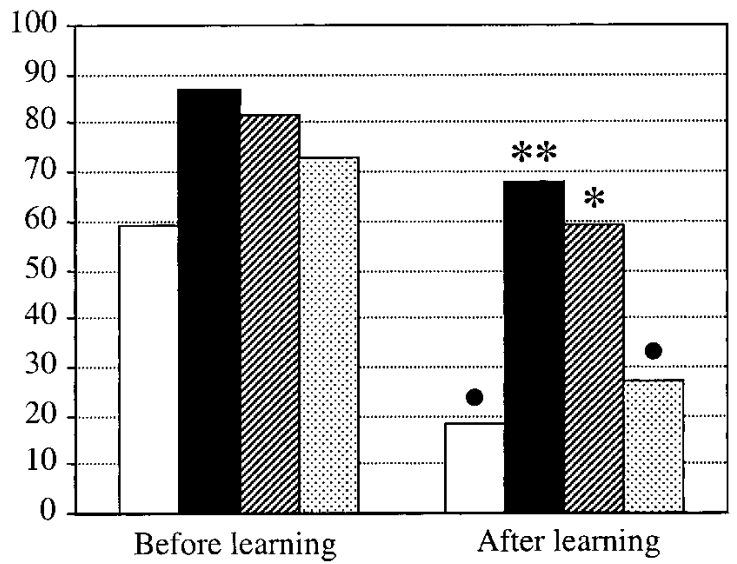

$\square \mathrm{aPC} \square \mathrm{pPC} \mathbb{Z} \mathrm{LEC} \cap \mathrm{DG}$

Figure 2 Effect of learning on late-component occurrence rate. (A) Occurrence rate (measured as described in Materials and Methods) was compared between control $(n=7)$ and trained $(n=11)$ groups after learning. (*) $P \leq 0.05$ ( $\chi 2$ comparisons): significant difference between trained and control animals in the corresponding recording site. $(B)$ Occurrence rate was compared between the different recording sites either before or after learning in animals of the trained group $(n=11)$. (*) $P<0.025,(* *) P<0.005$ (Fisher's test): significant difference with the anterior piriform cortex (aPC) and the dentate gyrus (DG) measured after learning. $(\bullet) P<0.005$ (Fisher's test): significant difference with aPC and DG measured before learning. ways significantly greater in pPC than in LEC $(P<0.05)$ for each subgroup.

\section{Learning Reveals a Second Late Component}

Observation of individual traces collected after learning in trained animals revealed the presence of a second late component (late component 2, LC2) with a mean latency of 140 msec (LC2; Fig. 3A). The rate of occurrence of the late component 2 was measured in the two experimental groups (control and trained) on signals collected during the second recording session (after learning). Chi-square comparisons revealed that the occurrence rate of this component was significantly increased in all the recorded sites of trained rats compared to control rats (Fig. 3B). Further comparisons in trained animals using Fisher's tests showed that, in contrast to what was observed for the first late component (late component 1), the rate of occurrence of late component 2 was similar in all four recording sites.

The amplitude and latency of late component 2 was measured in trained rats (Table 3). One-way ANOVA showed that the amplitude of late component 2 did not differ significantly between the recording sites $\left(F_{3,24}=2.01 ; P=0.14\right)$. Similarly, the latency was comparable in all four recording sites $\left(F_{3,24}=2.02 ; P=0.14\right)$.

In summary, late component 2 was observed almost exclusively in trained rats after learning. It occurred with the same amplitude and latency in all four recorded sites.

\section{DISCUSSION}

This study was aimed at investigating the consequences of learning on late polysynaptic components of EFP signals recorded in parallel at different levels of the olfactory pathways, namely aPC, pPC, LEC, and DG. The data confirmed previous observation that learning was associated with a lowering in late-component-1 optimal intensity of stimulation in pPC and extended our finding to additional brain areas. Specifically, whereas before learning, late component 1 was rather uniformly distributed among the recorded sites; following learning its expression was facilitated preferentially in pPC and LEC. Furthermore, learning was accompanied by the emergence of a new late component (late component 2), which occurred simultaneously in the four recording sites. These effects were independent of the learned significance of the stimulus because S+ and S- signals exhibited similar results.

\section{Are the Observed Changes Related to Learning?} Stripling et al. (1988) and Stripling and Patneau (1999) showed that repeated high frequency $(100 \mathrm{~Hz})$ stimulation of the granule cell layer of the olfactory bulb induced a selective potentiation of late components in field potentials evoked in piriform cortex. Although these results may suggest our effects are the result of repetitive OB stimulation

$$
\begin{array}{lllllllllllllll} 
& E & A & R & N & I & N & G & \mathcal{Z} & M & E & M & O & R & Y \\
\text { www.learnmem.org } & & &
\end{array}
$$


Table 2. Amplitude of Late Component in Control and Trained Animals in pPC and LEC

\begin{tabular}{|c|c|c|c|c|}
\hline \multirow[b]{2}{*}{ Structure } & \multicolumn{2}{|c|}{ Control group } & \multicolumn{2}{|c|}{ Conditioned group } \\
\hline & Before & After & Before & After \\
\hline Posterior part of piriform cortex & $453 \pm 53$ & $383 \pm 49$ & $629 \pm 79$ & $579 \pm 73$ \\
\hline Lateral entorhinal cortex & $114 \pm 20^{\mathrm{a}}$ & $108 \pm 18^{\mathrm{a}}$ & $275 \pm 75^{\mathrm{a}}$ & $269 \pm 33^{a}$ \\
\hline
\end{tabular}

${ }^{\text {a }} P<0.05$; significant difference with the value just above in the same column.

rather than learning, the performance of our control animals suggests otherwise. Specifically, our control group was exposed to the same $50-\mathrm{Hz}$ electrical stimulation as the trained animals but with no association to a reinforcement. Control animals exhibited no changes in late-component parameters. Therefore the above described effects can be ascribed to learning and not to nonspecific effects due to repeated electrical stimulation.

\section{Characteristics of Late vs. Early Component in Naive Rats}

The early component presents the lowest latency and the highest amplitude in aPC, followed by decreasing amplitude and increasing latency in more caudal sites. Concerning late component, prelearning recordings only detected late component 1 that occurred with the lowest latency and the greatest amplitude in pPC, suggesting the signal is generated in the pPC, followed by neighboring structures. These data confirm previous observations reported for piriform and entorhinal cortices (Mouly et al. 1998) and provide new information concerning late component occurrence in the dentate gyrus.

The origin of the early component is rather well understood for structures in the olfactory pathway. In PC and LEC, the early component is ascribed to the mono- and disynaptic depolarization of pyramidal cells (Van Groen et al. 1987; De Curtis et al. 1991; Ketchum and Haberly 1993). In the DG, the early component seems to reflect the polysynaptic activation of the granule cell dendrites (Wilson and Steward 1978; Habets et al. 1980). In contrast, there is less understanding for the origin of the late component. Our previous work showed that late component occurred in an all-or-none fashion and for a limited range of low intensities of stimulation of the olfactory bulb. Indeed, as stimulation intensity increased, the late component disappeared (Mouly et al. 1998). In the piriform cortex, other studies have described the occurrence of late component in vivo (Ferreyra Moyano et al. 1984, 1988; Stripling et al. 1988; Stripling and Patneau 1999) or in vitro (Chujo 1978; Tseng and Haberly 1989a,b; Sugitani et al. 1994) in the rat. Working on piriform cortex slices, Tseng and Haberly (1989a,b) and Hoffman and Haberly (1991) found that the late excitatory postsynaptic potentials (EPSPs) recorded in superficial pyramidal cells in response to fiber-tract stimulation were in fact synap- tically driven from deep cells mainly concentrated in the endopiriform nucleus, a region subjacent to layer III of the PCx. In a recent work, Behan and Haberly (1999) showed that the endopiriform nucleus and the piriform cortex send projections to the same target areas. This led the investigators to propose that synchronous discharge in the endopiriform nucleus could generate depolarization of pyramidal cells throughout the piriform cortex and other olfactory cortical areas. In a previous study (Mouly et al. 1998), we confirmed that the late component was actually observed in the piriform cortex and the entorhinal cortex but not in the olfactory bulb. In our present study, we show that the late component also spreads to the dentate gyrus, where it occurs with the longest latency. Taken together, these data are in accordance with the described efferent connections of the endopiriform nucleus (Behan and Haberly 1999).

The comparison between early- and late-component characteristics and their possible origins leads us to propose that these components are the result of two different networks that can be revealed by the use of low- or highstimulation intensities. Both networks share in common the activation of pyramidal cells. The network involved in latecomponent generation is triggered by low intensities of stimulation. It could mainly include the activation of deep cells in endopiriform nucleus in response to moderate depolarization of piriform cortex pyramidal cells. With high intensities of stimulation, the depolarization of pyramidal cells is stronger and the activation of deep multipolar cells could be short-circuited via the recruitment of local inhibitory interneurons. Such a dependence of late-excitatoryevents occurrence on intensity of stimulation has recently been described in perirhinal cortex neurons in the isolated guinea pig brain (Biella et al. 2001).

\section{Effects of Learning on Late Components}

Learning resulted in a lowering of the first late-component optimal intensity as measured in pPC. Indeed, following learning, late component occurred at intensities lower than those required in prelearning animals. Moreover, at these low intensities, the early component was almost absent. In contrast to optimal intensity lowering, late-component amplitude was not modified by learning. This further supports the assumption that the late component is an all-or-none rather than incremental phenomenon: Once the conditions

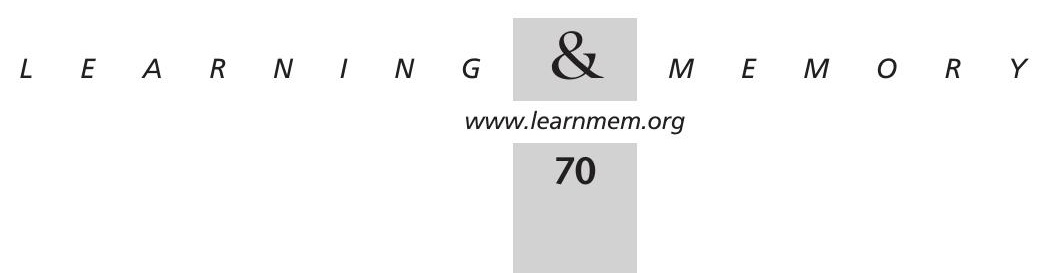


A
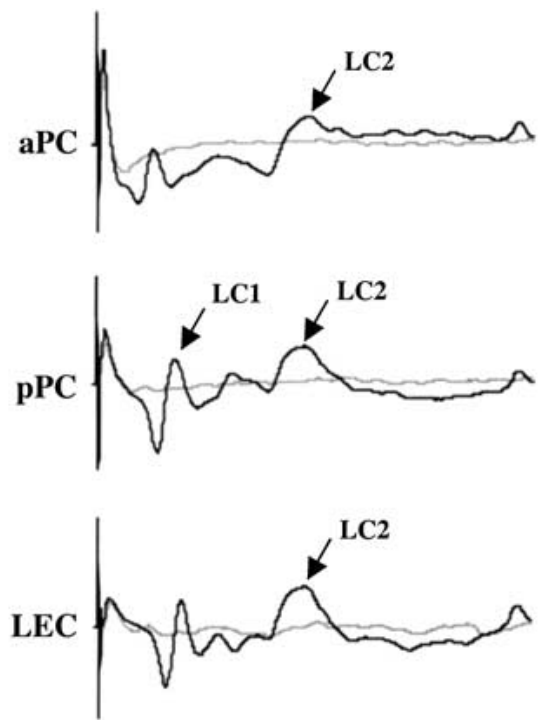

$0.5 \mathrm{mV}$

$50 \mathrm{~ms}$

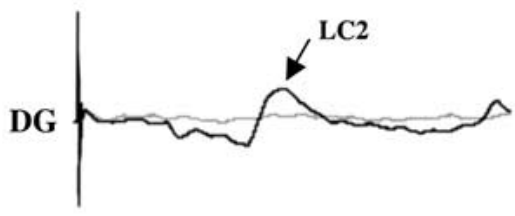

B

\section{Occurrence rate}

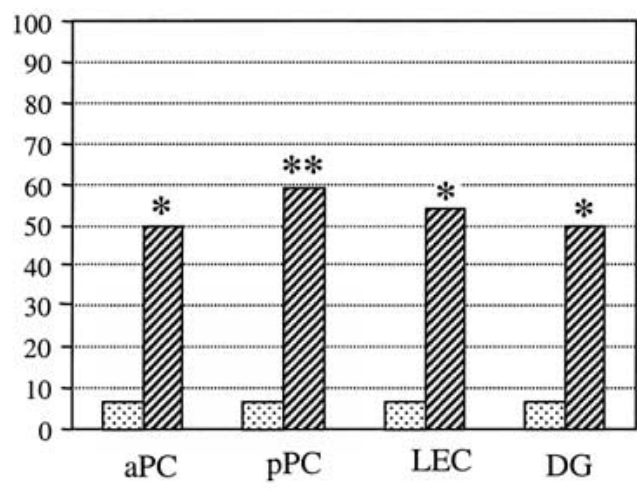

Control group $\mathbb{Z}$ Trained group

Figure 3 Effect of learning on the facilitation of a second late component. (A) An example of individual evoked field potentials (traces are averages of 12 sweeps) collected in a trained rat in the different sites before (light grey line) and after (dark line) learning for the same intensity of stimulation of the olfactory bulb. Following learning, a second late component (LC2; mean latency: 150 msec) was observed in all the recorded sites. (B) LC2 occurrence was compared between control $(n=7)$ and trained $(n=11)$ animals after learning. (*) $P<0.01,(* *) P<0.005$ ( $\chi^{2}$ comparisons): significant difference between trained and control animals in the corresponding recording site. of its induction are fulfilled, it occurs with a stable amplitude (Mouly et al. 1998).

Based on these results, we hypothesized that learning has altered synaptic strengths between network elements responsible for the generation of the late component. Because the late component was observed in the quasi absence of a measurable early component, it can be suggested that learning has potentiated transmission within the latecomponent network. Specifically, perhaps learning strengthened existing excitatory intrinsic connections between multipolar cells of the endopiriform nucleus (Hoffman and Haberly 1993; Behan and Haberly 1999).

Before learning, late component was induced rather uniformly (albeit with different amplitudes) in all four recorded sites. However, following learning its occurrence at low intensity was specifically facilitated in PPC and LEC. In previous work using voltage-sensitive dye to map piriform cortex activity, we showed that learning greatly enhanced the occurrence of the late component in posterior piriform cortex (Litaudon et al. 1997). In our present study, the use of parallel recordings at several levels of the olfactory pathways extend our findings to the distribution of the late component in the network. Indeed, the present data indicate that the facilitation of the late component was not restricted to $\mathrm{pPC}$, but occurred also in LEC; whereas it was not observed in the aPC nor in the DG. These data further confirm the existence of a functional dissociation between anterior and posterior piriform cortex with respect to mnesic processes (Mouly et al. 2001) and point to a similar involvement of pPC and LEC in this learning paradigm.

Our data do not confirm those of Chaillan et al. (1999) who reported potentiation of a polysynaptic response in the DG during learning. However, it is important to note that in our study, a recording session began $1 \mathrm{~h}$ after a training session; whereas Chaillan et al. (1999) recordings were done immediately after the training session. In the latter study, the observed potentiation was maximal just after each training session and decreased to a lower level when measured $24 \mathrm{~h}$ later. Therefore, the difference in the recording paradigm could explain the discrepancy in the data obtained in these two studies.

In addition to lowering the optimal intensity of the first late component, learning also revealed the presence of a new late component at longer latency (late component 2). In contrast to late component 1 , which occurred preferentially in pPC and LEC following learning, late component 2 was detected in all four recording sites, where it presented the same amplitude and latency. Whether late component 2 is secondary to the facilitation of late component 1 or reflects the recruitment of another polysynaptic circuit cannot be determined based on our data. Chapman et al. (1998) and Trepel and Racine (1998) have shown that repeated daily tetanization of the corpus callosum induces lasting changes in sensorimotor cortex field potential responses,

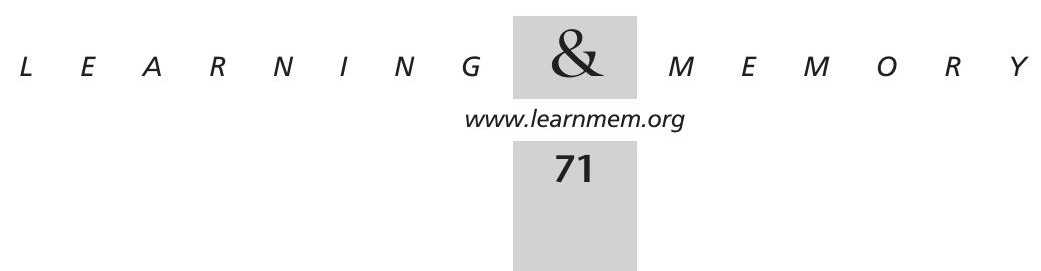


Table 3. Amplitude and Latency of Late Component 2 in Trained Animals in the Different Recording Sites

\begin{tabular}{lcccc}
\hline & \multicolumn{4}{c}{ Structure } \\
\cline { 2 - 5 } Parameter & $\begin{array}{l}\text { Anterior part of } \\
\text { piriform cortex }\end{array}$ & $\begin{array}{l}\text { Posterior part of } \\
\text { piriform cortex }\end{array}$ & $\begin{array}{c}\text { Lateral entorhinal } \\
\text { cortex }\end{array}$ & $\begin{array}{c}\text { Dentate } \\
\text { gyrus }\end{array}$ \\
\hline Amplitude $(\mu \mathrm{V})$ & $354 \pm 61$ & $272 \pm 38$ & $296 \pm 34$ & $290 \pm 17$ \\
Latency $(\mathrm{msec})$ & $146 \pm 8$ & $143 \pm 8$ & $144 \pm 8$ & $147 \pm 8$ \\
\hline
\end{tabular}

among which was the emergence of a new late component. The investigators proposed that potentiation of intracortical horizontal connections could have contributed to the observed facilitation of new late components. The same assumption could be made in our study.

\section{Functional Interpretations}

In prelearning animals, late component 1 was observed in parallel at different levels of the olfactory pathways and was induced in response to low-intensity electrical stimulation of the olfactory bulb. It reflects a massive synchronous depolarization of underlying principal cells 50-60 msec after the arrival of a stimulus. If this phenomenon occurs in response to a natural olfactory stimulus, these data could suggest how odors of low concentration are processed within the olfactory pathways. Furthermore, in preliminary experiments we observed that in waking animals, the late-component occurrence seems to be gated by the animal's vigilance state (A.M. Mouly and R. Gervais, unpubl. data). Specifically, the late component is observed during periods of quiet immobility or sleep, thus suggesting preferential neural processing during these particular states of brain activity. Because the olfactory bulb appears to be engaged in posttraining consolidation processes (Mouly et al. 1993), one could speculate that during sleep episodes that follow a training session, the bulbar neurons recently activated by the to-belearned stimulus maintain a low level of excitation. This might enable the occurrence of synchronous population discharges in the endopiriform nucleus, which in turn generate depolarization of neuronal populations in the different projection sites. As already proposed by Behan and Haberly (1999) and by analogy with the postulated function of sharp waves in the hippocampus (Buzsaki 1996), such synchronous depolarizations might enable synaptic changes (through the activation of NMDA receptors) at the appropriate nodes of the network, thus participating in the laying down of memory. Theses changes would be responsible for the observed lowering of the late-component-1 optimal intensity in our trained animals and for the emergence of the late component 2 , which could reflect reverberating or oscillatory activity within the consolidated network. Recording in parallel at several levels of the olfactory pathways allowed us to pinpoint the differential involvement of the investigated areas in these processes.

\section{MATERIALS AND METHODS}

\section{Animals}

Eighteen male Wistar rats (280-300 $\mathrm{g}$ at the time of surgery) purchased from IFFA-CREDO were used in this experiment. These animals participated in a previous experiment (Mouly et al. 2001). They were housed individually in clear cages with food and water freely available. During training, access to water was limited to 30 min following each daily session. In strict accordance with French and European guidelines for animal experimentation, all efforts were made to minimize the number of animals used, as well as their suffering.

\section{Electrode Surgery}

The animals were anaesthetized with Equithesin (a mixture of chloral hydrate and sodium pentobarbital; $3 \mathrm{~mL} / \mathrm{kg}$, I.P.). The level of anesthesia was held constant with regular injections of Equithesin throughout surgery. The animals were fixed in a stereotaxic apparatus with the head flat, and holes were drilled for implantation of two bipolar stimulating electrodes in the left $\mathrm{OB}$ (electrode OB1: $\mathrm{A} / \mathrm{P}-5.5 \mathrm{~mm}$ relative to the nasofrontal suture, $\mathrm{L} 1.3 \mathrm{~mm}$ relative to bregma; electrode $\mathrm{OB} 2: \mathrm{A} / \mathrm{P}-7 \mathrm{~mm}$ relative to the nasofrontal suture, $\mathrm{L} 1.3 \mathrm{~mm}$ relative to bregma) and four monopolar recording electrodes positioned, respectively, in the aPC $(\mathrm{A} / \mathrm{P}+2.2 \mathrm{~mm}, \mathrm{~L} 4$ $\mathrm{mm}$ relative to bregma), the $\mathrm{pPC}(\mathrm{A} / \mathrm{P}-2.3 \mathrm{~mm}, \mathrm{~L} 5.4 \mathrm{~mm}$ to bregma), the DG (A/P $-4.2 \mathrm{~mm}$, L $3 \mathrm{~mm}$ relative to bregma) and the LEC (A/P $-6.3 \mathrm{~mm}$, L $6 \mathrm{~mm}$ relative to bregma) (Fig. 4A).

The bipolar stimulating electrodes consisted of two 100- $\mu \mathrm{m}$ stainless-steel wires (California Fine Wire) with a tip separation of $500 \mu \mathrm{m}$ in depth. The recording electrodes consisted of single $100-\mu \mathrm{m}$ stainless-steel wires. The depth of the stimulating electrodes was adjusted at the level of mitral cell layer using electrophysiological monitoring of the characteristic large multiunit mitral cell activity. Accurate positioning of recording electrodes depth was achieved using the field potential profile evoked in each structure in response to electrical stimulation of the bipolar $\mathrm{OB}$ electrodes (Fig. 4B). In aPC, pPC, and LEC, recording electrode tips were positioned in the deep cortical layers (layer III) where the field potential signal presented a large, stable amplitude, which corresponded to the approximate depths of $-7 \mathrm{~mm},-8 \mathrm{~mm}$, and $-6.5 \mathrm{~mm}$, respectively. In DG, the recording electrode tip was positioned at the level of the granular layer, at an approximate depth of $3 \mathrm{~mm}$. One skull screw placed above the contralateral parietal cortex served as a ground and reference electrode for monopolar field potential recordings. The electrode leads were connected to a 9-pin connector and the assembly was embedded in

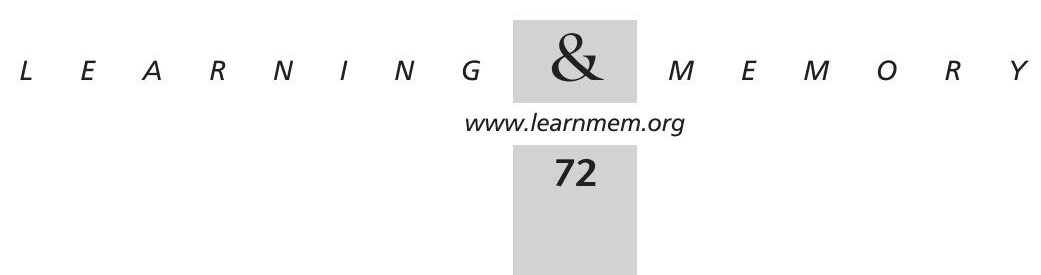




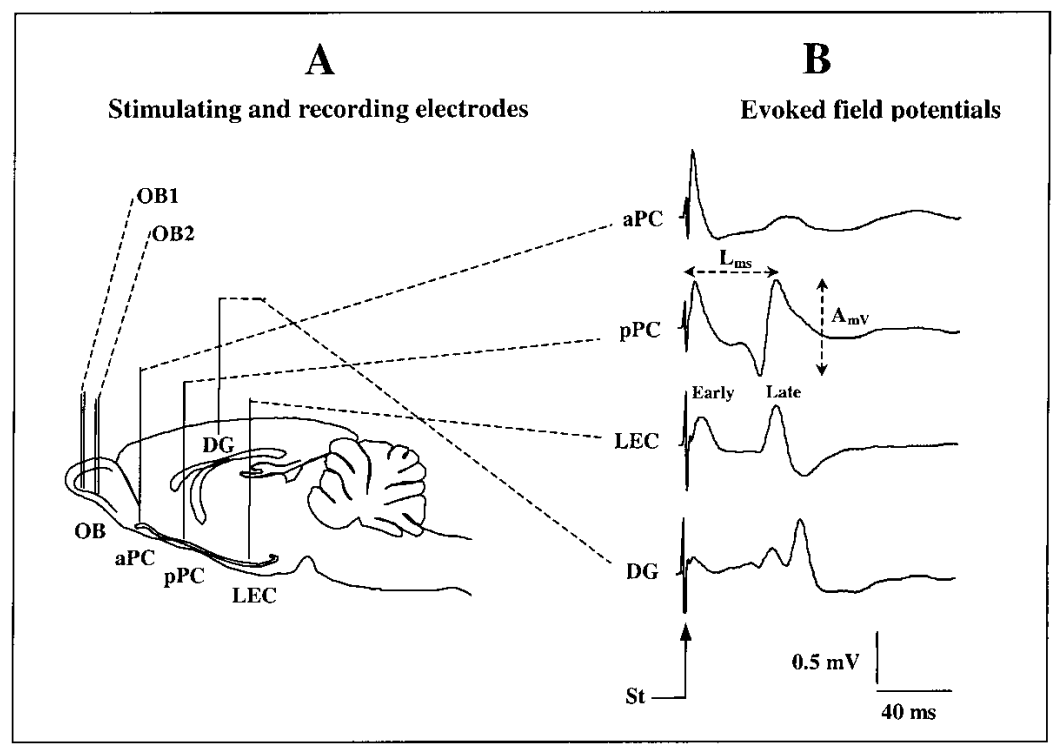

Figure 4 Schematic representation of the implanted electrodes and recorded signals. (A) Two bipolar stimulating electrodes (OB1 and OB2) were implanted in the ventral mitral cell layer of the olfactory bulb (OB). Four monopolar recording electrodes were implanted, respectively, in the anterior piriform cortex (aPC), posterior piriform cortex (pPC), lateral entorhinal cortex (LEC), and dentate gyrus (DG). (B) An example of the evoked field potential signals induced in the four recording sites in response to stimulation of the OB. The amplitude $\left(\mathrm{A}_{\mathrm{mv}}\right)$ and latency $\left(\mathrm{L}_{\mathrm{ms}}\right)$ of the evoked potential late component were measured in each site, as indicated on the figure. $(\mathrm{St}$,$) stimulation$ artifact.

dental cement. The animals were allowed 2 wk of postsurgical recovery.

\section{Behavioral Discrimination Task}

Rats were required to learn to associate electrical stimulation of one OB electrode (for example, OB1) with sucrose $(40 \mathrm{~g} / \mathrm{L})$ and electrical stimulation of the other OB electrode (for example, OB2, counterbalanced between animals) with quinine $(0.5 \mathrm{~g} / \mathrm{L})$. The electrical stimulation consisted in a sine wave $(50 \mathrm{~Hz}$ in frequency) at an intensity individualized for each rat based on the intensity required to induce sniffing. The resulting values ranged between 7 and $10 \mu \mathrm{A}$. Animals respond to this electrical stimulation in a manner similar to natural odor delivery with behaviors such as sniffing, exploration, and rearing.

The experimental setup and the training procedure have been described in detail elsewhere (Mouly et al. 2001). Briefly, the task was performed in a Plexiglas cage containing two drinking ports that became accessible to the animal approximately every $2 \mathrm{~min}$. The first three daily sessions (familiarization sessions) began with the water-deprived animals being familiarized with the apparatus by providing access to both drinking ports without the preceding stimulation of the OB. Subsequently, the animal was given access to the drinking ports with $\mathrm{OB}$ electrical stimulation automatically triggered when the animal interrupted a photobeam placed $2 \mathrm{~cm}$ in front of each drinking outlet. At this point, the animal could choose to continue approaching the waterspout or withdraw from it and approach the other waterspout. An animal was considered to have made a choice when it licked one of the waterspouts, which initiated the delivery of the corresponding solution. OB stimulation lasted as long as the rat's head interrupted the photobeam, inde- pendent of whether or not the animal was drinking the solution. A choice was scored as correct when the animal licked the sucrose water outlet. In this case the rat was given access to the sucrose solution for $20 \mathrm{sec}$. Licking the quinine spout was considered an incorrect choice and the rat was negatively reinforced with a drop of quinine solution. The solution-delivery system was constructed to eliminate possible olfactory cues from the sucrose and quinine solutions, forcing the animal to depend upon the OB stimulation cues. Additionally, the position of the sucrose and quinine spouts were randomized from trial to trial to prevent the animal from using position cues. Each daily session was followed by providing the animals with free access to water for $30 \mathrm{~min}$ in a holding cage. Learning was considered complete when the animal made at least 16 correct choices out of the 20 trials $(80 \%)$ within a session on 2 consecutive sessions. Animals generally reached learning criterion within only 3 to 5 sessions, with animals receiving 1 session per day.

\section{Experimental Groups}

Two groups of animals were used: a trained group and a control group. In the trained group $(n=11)$ the rats were conditioned as described above. For 6 of the 11 animals, sucrose was signaled by stimulation of electrode $\mathrm{OB} 1$ and quinine by stimulation of electrode $\mathrm{OB} 2$, and for the remaining 5 rats it was the reverse. In the control group $(n=7)$ the animals underwent the same protocol of electrical stimulation as that experienced in the trained group but without access to either quinine or sucrose. Indeed, during a pseudotraining session, 20 trials were performed during which electrical stimulation onset was triggered by the experimenter. In each trial, the duration of electrical stimulation through one OB electrode was similar to that received by a trained rat drinking sucrose $(20 \mathrm{sec})$, whereas the duration of stimulation through the other electrode was similar to that received by a trained rat drinking quinine (2-3 sec). The intensity of stimulation was individualized for each control rat based on the intensity required to induce sniffing. After each session, the rats had free access to water for $30 \mathrm{~min}$.

\section{Recording Procedure}

Recordings were done before and after learning in each animal. In previous experiments, we observed that late-component occurrence was greatly facilitated under anesthesia (A.M. Mouly and R. Gervais, unpubl. data), therefore electrophysiological recordings were carried out on anesthetized animals. The first recording session was carried out $1 \mathrm{~h}$ after the first familiarization session and constituted the anesthetized baseline response. The second recording session was done $1 \mathrm{~h}$ after the last training session (criterion reached). The animal was anesthetized with a single injection of Equithesin $(3 \mathrm{~mL} / \mathrm{kg}$, I.P.) and placed in a round Plexiglas cage that is markedly different from the one used during conditioning. Recording and stimulating cables were relayed at the top of the cage through a multichannel swiveling electrical connector. The recording cable contained a five-channel JFET headstage.

Electrical stimulation that was used to induce evoked field

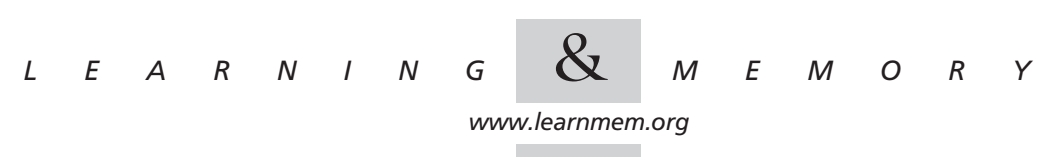


potentials (EFPs) was delivered through a Master-8 stimulator (AMPI) and a photically isolated constant current unit. The electrical stimulus was a single monophasic square pulse $0.1 \mathrm{msec}$ in duration and $0.1 \mathrm{~Hz}$ in frequency. For each animal, the optimal intensity of stimulation for inducing the late component was determined. Each acquisition episode consisted of collecting 12 sweeps in parallel through the four recording electrodes in response to stimulation of each bulbar electrode (OB1 and OB2). For the sake of brevity, we will note the S+ signals as the EFPs induced by stimulation of the $\mathrm{OB}$ electrode associated with the delivery of sucrose during training sessions, and the S- signals as the EFPs induced by stimulation of the $\mathrm{OB}$ electrode signaling the delivery of quinine.

The signals induced in the four recording sites (Fig. 4B) in response to $\mathrm{OB}$ stimulation were amplified (Grass Model 12, AstroMed), filtered (1-300 $\mathrm{Hz}$ ), and digitized (sampling frequency: 5 $\mathrm{kHz}$ ) using a data-acquisition system (Wavebook 512, Iotech) for storage on computer hard disk.

\section{Data Analysis}

Off-line, individual EFPs were averaged and analyzed using Dasylab data-acquisition software (Iotech).

In a previous study (Mouly et al. 1998) the late component was shown to present the following characteristics. It is an all-ornone phenomenon (i.e., it is not systematically present on individual signals, but when present its amplitude is large and stable). It is induced for a very limited range of low intensities of stimulation, within which it is tuned to an optimal intensity, defined as the intensity at which its frequency of occurrence among the sampled traces is maximal.

In our present study, the late component was therefore characterized according to three criterion. First, its optimal intensity was determined in the pPC signals as the intensity for which late component was observed in at least 8 sweeps of the 12 sampled for further averaging. Detection of late component on individual sweeps was easily achieved due to its all-or-none character. The amplitude threshold for assessing the presence of the late component in pPC signals was set to $100 \mu \mathrm{V}$, which represents twice the baseline spontaneous variability in this site. The latecomponent optimal intensity was measured in the pPC signals, compared between the two recording sessions using two-way (learning $\times$ reinforcement) ANOVA for repeated measures, and then followed by Wilcoxon matched-pairs signed-ranks tests.

Second, for each recording site, the rate of occurrence of the late component was determined by the percentage of signals on which a late component was observed. For example a rate of occurrence of $80 \%$ in pPC indicated that the late component was detected in $80 \%$ of the mean EFP signals collected in the pPC. The obtained values were compared either between the recording sites of a given group using Fisher's exact test or between the two groups for a given site using $\chi^{2}$ comparisons.

Third, the amplitude and latency of the late component were measured on mean signals in each recording site. Mean signals were obtained by averaging individual traces on which a late component was observed. The number of averaged traces could therefore vary from 8 to 12 . Late-component amplitude was measured from peak to peak, as indicated in Figure 4B. Late-component latency was determined as the latency to positive peak from the stimulus artifact. The obtained values were compared using oneway ANOVA for repeated measures, followed by Wilcoxon matched-pairs signed-ranks tests. For all the statistical comparisons performed, the significance level was set at 0.05 .

\section{Histology}

At the end of the experiment, the rats were deeply anaesthetized and perfused intracardially with a $0.9 \%$ saline solution, followed by a $10 \%$ formalin solution. The brains were dissected, stored in formalin for $1 \mathrm{wk}$, cut into $40-\mu \mathrm{m}$ slices, and stained with cresyl violet, and the positions of recording and stimulating electrodes were verified.

\section{ACKNOWLEDGMENTS}

We gratefully acknowledge Michel Vigouroux, Vincent Farget, Bernard Bertrand, and Belkacem Messaoudi for their technical support and Alexandra Fort and Nadia Benboutayab for their help during the experiments. We also thank Dr. Regina Sullivan for a careful review of the English. This work was supported by the CNRS.

The publication costs of this article were defrayed in part by payment of page charges. This article must therefore be hereby marked "advertisement" in accordance with 18 USC section 1734 solely to indicate this fact.

\section{REFERENCES}

Behan, M. and Haberly, L.B. 1999. Intrinsic and efferent connections of the endopiriform nucleus in rat. J. Comp. Neurol. 408: 234-548.

Biella, G., Uva, L., and De Curtis, M. 2001. Network activity evoked by neocortical stimulation in area 36 of the guinea pig perirhinal cortex. J. Neurophysiol. 86: 164-172.

Buzsaki, G. 1996. The hippocampo-neocortical dialogue. Cereb. Cortex 6: 281-292.

Chaillan, F., Truchet, B., Roman, F.S., and Soumireu-Mourat, B. 1999. Early polysynaptic potentiation recorded in the dentate gyrus during an associative learning task. Neuroscience 94: 443-451.

Chapman, C.A., Trepel, C., Ivanco, T.L., Froc, D.J., Wilson, K., and Racine, R.J. 1998. Changes in field potentials and membrane currents in rat sensorimotor cortex following repeated tetanization of the corpus callosus in vivo. Cereb. Cortex 8: 730-742.

Chujo, T. 1978. Neuronal mechanisms of the late $\mathrm{N}$-wave induced in vitro in thin sections of the olfactory cortex of rats. Jap. J. Physiol. 28: $433-445$.

De Curtis, M., Paré, D., and Llinas, R. 1991. The electrophysiology of the olfactory-hippocampal circuit in the isolated and perfused adult mammalian brain in vitro. Hippocampus 1: 341-354.

Ferreyra Moyano, H., Cinelli, A.R., and Molina, J.C. 1984. Origin and plastic properties of late components evoked in the rat's but not in the armadillo's prepyriform cortex. Neurosci. Lett. 52: 305-310.

Ferreyra Moyano, H., Cinelli, A.R., Molina, J.C., and Barragan, E. 1988. Current generators and properties of late component evoked in rat olfactory cortex. Brain Res. Bull. 20: 433-446.

Habets, A.M.M.C., Lopez Da Silva, F.H., and Mollevanger, W.J. 1980. An olfactory input to the hippocampus of the cat: Field potential analysis. Brain Res. 182: 47-64.

Hoffman, W.H. and Haberly, L.B. 1991. Bursting-induced epileptiform EPSPs in slices of piriform cortex are generated by deep cells. $J$. Neurosci. 11: 2021-2031.

- 1993. Role of synaptic excitation in the generation of bursting-induced epileptiform potentials in the endopiriform nucleus and piriform cortex. J. Neurophysiol. 70: 2550-2561.

Ketchum, K.L. and Haberly, L.B. 1993. Membrane currents evoked by afferent fiber stimulation in rat piriform cortex. I. Current source-density analysis. J. Neurophysiol. 69: 248-260.

Litaudon, P., Mouly, A.M., Sullivan, R., Gervais, R., and Cattarelli, M. 1997. Learning-induced changes in rat piriform cortex activity mapped using multisite recording with voltage sensitive dye. Eur. J. Neurosci. 9: 1593-1602.

Martin, S.J., Grimwood, P.D., and Morris, R.G.M. 2000. Synaptic plasticity and memory: An evaluation of the hypothesis. Annu. Rev. Neurosci. 23: 649-711.

\section{…………....}


Mouly, A.M., Vigouroux, M., and Holley, A. 1985. On the ability of rats to discriminate between microstimulation of the olfactory bulb in different locations. Behav. Brain Res. 17: 45-48.

Mouly, A.M., Kindermann, U., Gervais, R., and Holley, A. 1993. Involvement of the olfactory bulb in consolidation processes associated with long-term memory in rats. Behav. Neurosci. 107: 451-457.

Mouly, A.M., Litaudon, P., Chabaud, P., Ravel, N., and Gervais, R. 1998. Spatiotemporal distribution of a late synchronized activity in olfactory pathways following stimulation of the olfactory bulb in rats. Eur. J. Neurosci. 10: 1128-1135.

Mouly, A.M., Fort, A., Ben-Boutayab, N., and Gervais, R. 2001. Olfactory learning induces differential long-lasting changes in rat central olfactory pathways. Neuroscience 102: 11-21.

Otto, T. and Eichenbaum, H. 1992. Olfactory learning and memory in the rat: A "model system" for studies of the neurobiology of memory. In The science of olfaction (eds. M. Serby and K. Chobor), pp. 213-244. Springer-Verlag, New York.

Slotnick, B. 2001. Animal cognition and the rat olfactory system. Trends Cogn. Sci. 5: 216-222.

Stripling, J.S. and Patneau, D.K. 1999. Potentiation of late components in olfactory bulb and piriform cortex requires activation of cortical association fibers. Brain Res. 841: 27-42.
Stripling, J.S., Patneau, D.K., and Gramlich, C.A. 1988. Selective long-term potentiation in the piriform cortex. Brain Res. 441: 281-291.

Sugitani, M., Sugai, T., Tanifuji, M., Murase, K., and Onoda, N. 1994. Optical imaging of the in vitro guinea pig piriform cortex activity using a voltage-sensitive dye. Neurosci. Lett. 165: 215-218.

Trepel, C. and Racine, R.J. 1998. Long-term potentiation in the neocortex of the adult, freely moving rat. Cereb. Cortex 8: 719-729.

Tseng, G.F. and Haberly, L.B. 1989a. Deep neurons in piriform cortex. I. Morphology and synaptically evoked responses including a unique high amplitude paired shock facilitation. J. Neurophysiol. 62: 369-385.

. 1989 b. Deep neurons in piriform cortex. II. Membrane properties that underlie unusual synaptic responses. J. Neurophysiol. 62: 386-400.

Van Groen, T., Lopes Da Silva, F.H., and Wadman, W.J. 1987. Synaptic organization of olfactory inputs and local circuits in the entorhinal cortex: A current source density analysis in the cat. Exp. Brain Res 67: 615-622.

Wilson, R.C. and Steward, O. 1978. Polysynaptic activation of the dentate gyrus of the hippocampal formation: An olfactory input via the lateral entorhinal cortex. Exp. Brain Res. 33: 23-534.

Received November 26, 2001; accepted in revised form March 19, 2002.

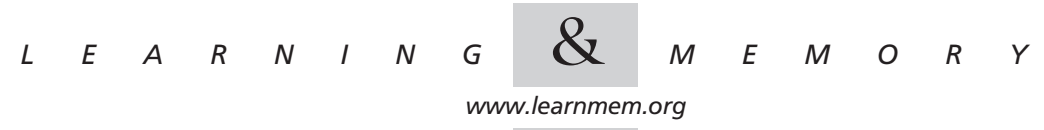




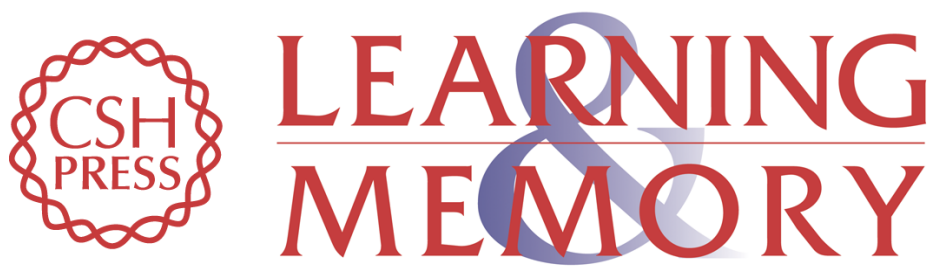

\section{Polysynaptic Potentiation at Different Levels of Rat Olfactory Pathways Following Learning}

Anne Marie Mouly and Rémi Gervais

Learn. Mem. 2002, 9:

Access the most recent version at doi:10.1101/lm.45602

References This article cites 27 articles, 1 of which can be accessed free at: http://learnmem.cshlp.org/content/9/2/66.full.html\#ref-list-1

License

Email Alerting Receive free email alerts when new articles cite this article - sign up in the box at the Service top right corner of the article or click here. 